\title{
A Component-Based WebGIS Geo-Union
}

\author{
Yingwei Luo, Xiaolin Wang, and Zhuoqun Xu \\ Dept. of Computer Science and Technology, Peking University, Beijing, P.R.China, 100871 \\ lyw@pku.edu.cn
}

\begin{abstract}
A component-based WebGIS system Geo-Union is introduced, including its architecture, functional partition and web application mode.
\end{abstract}

\section{Introduction}

Assigning functions in reason and improving performance are two key issues for making WebGIS more practicable [1]. So based on analyzing modeling technique of component-based WebGIS, we construct a practicable, multi-level WebGIS system.

\section{A Component-Based WebGIS: Geo-Union}

Geo-Union has a four-level architecture: application layer, component layer, service layer and storage layer, where service layer has different units to provide both client services and server services. Figure 1 shows the architecture [2].

(1) Storage layer is the ground of Geo-Union. Storage layer is responsible for storage and management of both spatial data and non-spatial data based on ORDB. (2) Service layer is in charge of spatial data access and process, which can be divided into another two parts: Geo-Union client provides data access and process services to component layer, and Geo-Union server provides data access and process services to Geo-Union client through interacting with storage layer. Geo-Union server can manage different spatial data resources, and also can reply to different spatial data requests from different clients. (3) Component layer provides a rich set of services (components) to develop domain-oriented GIS applications for further developers. Component layer provides interface of GIS functions to users, but the implementation details are completed in service layer. (4) Application layer is to exploit application systems for different domains by assembling and integrating Geo-Union components.

Most of functions are implemented in service layer. Geo-Union server side is just to provide spatial data access. But Geo-Union client supports to develop domainoriented applications. In order to provide flexible developing mode, around map visualization objects, we designed another six types of objects: spatial data access objects, map edit objects, spatial analysis objects, mouse tool objects, utility objects 
and AppTool. Figure 2 shows the architecture of service layer, the roles of different objects and their relationships [2]. Figure 3 shows the Web application mode.

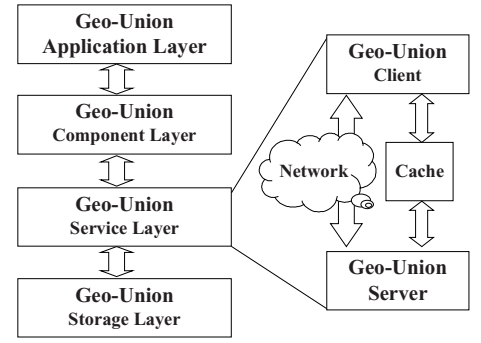

Fig. 1. Geo-Union Architecture

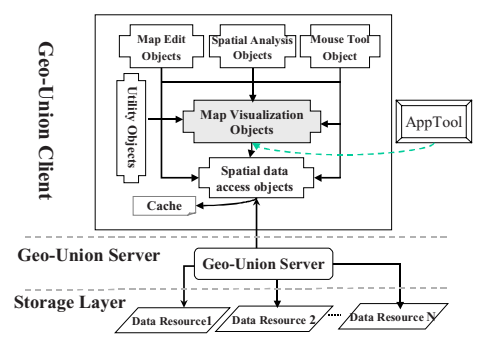

Fig. 2. Functional Partition of Service Layer

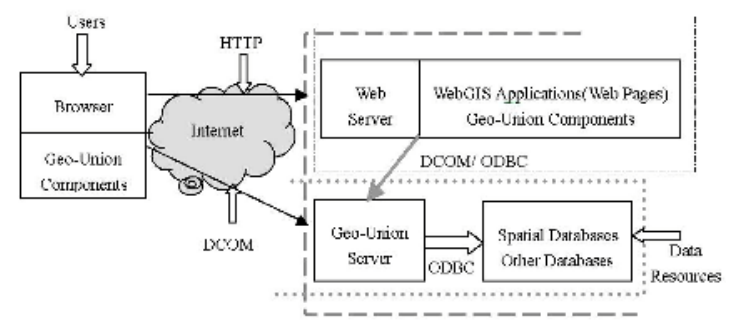

Fig. 3. Web Application of Geo-Union

\section{Conclusion}

Geo-Union has finished a preliminary component-based model for distributed WebGIS, and has got into use in many fields with sound effects.

Acknowledgements. This work is supported by the National Research Foundation for the Doctoral Program of Higher Education of China (20020001015); the National Grand Fundamental Research 973 Program of China (2002CB312000); the National Science Foundation of China (60203002); the National High Technology Development 863 Program (2002AA135330); the Beijing Science Foundation (4012007).

\section{References}

1. Li Bin: A Component Perspective on Geographic Information Services, Cartography and Geographic Information Science, 27(1): 75-86(2001).

2. Dept. of Computer Science and Technology, Peking University: Operation and Component Guide for Geo-Union Enterprise (in Chinese), Technology Material, http://gis.pku.edu.cn. 